\section{Acampe por una justicia ecológica: una experiencia de extensión y acceso a la justicia en la localidad de Santo Domingo, provincia de Santa Fe}

\author{
Cintia Giuliana Balaudo \\ cintibalaudo@live.com \\ (iD) orcid.org/0000-0001-6480-3222 \\ Dabel Leandro Franco \\ dabel.leandro@gmail.com \\ (iD) orcid.org/0000-0001-6693-3629 \\ María Virginia Gazzo \\ mvirginiag20@gmail.com \\ (iD) orcid.org/0000-0001-5363-0178
}

RECEPCIÓN: 11/05/21

ACEPTACIÓN FINAL: 18/08/21

\section{Resumen}

Desde el año 2019 se desarrolla en el ámbito de la Facultad de Ciencias Jurídicas y Sociales de la Universidad Nacional del Litoral el Proyecto de Extensión de Interés Social "Acampe por una justicia ecológica". El mismo considera especialmente la dimensión territorial con miras a identificar desigualdades en el acceso a la justicia en materia ambiental. Para ello y mediante la realización de campamentos jurídicos, se toma contacto con personas que residen en pequeños pueblos y ciudades agrícolas de la provincia de Santa Fe y se analiza el estado de situación de la problemática ambiental y los mecanismos existentes para peticionar en defensa de derechos vulnerados. En esta oportunidad, se aborda la experiencia transcurrida en la localidad de Santo Domingo, ubicada en el departamento Las Colonias. Se relatan las tareas llevadas a cabo y se destacan los resultados obtenidos en las encuestas, entrevistas y actividades realizadas.

Palabras clave: acceso a la justicia; acampes; derecho ambiental; riesgos socioambientales; conflictos socioambientales.

\author{
Angelina Rodríguez \\ angelina_r14@hotmail.com \\ (iD) orcid.org/0000-0003-0800-9002 \\ Universidad Nacional del Litoral, Argentina.
}

Camp for ecological justice: an experience of extension and access to justice in the town of Santo Domingo, Province of Santa $\mathrm{Fe}$

\section{Abstract}

A Social Interest Extension Project, called "Social "Acampe por una justicia ecológica", from the Law School at Universidad Nacional del Litoral has been developed since the second half of 2019. It deals with the access to justice in environmental issues and considers particularly the local dimension in order to identify inequalities of the access. For this, and through camping, contact is made with people who reside in small towns and agricultural cities in the province of Santa Fe with de object of analyce the state of the environmental problem and the existing mechanisms to petition in defense of violated rights. On this occasion, the experience took place in the town of Santo Domingo, located in the Las Colonias Department. The tasks carried out are reported, highlighting the results obtained in the surveys, interviews and activities carried out during the camp.

Keywords: access to justice; camps; environmental rights; social-environmental risks; social-environmental issues.
Ambiente y extensión universitaria /

Intervenciones

\author{
Acampamento por uma justiça ecológica: \\ uma experiência de extensão e acesso à \\ justiça no município de Santo Domingo, \\ província de Santa Fe
}

\section{Resumo}

Desde 2019, na Faculdade de Ciências Jurídicas e Sociais da Universidade Nacional del Litoral, desenvolve-se tem se desenvolvido o projeto de extensão de interesse social nomeado "Acampamento por uma justiça ecológica”. Considera especialmente a dimensão territorial para identificar as desigualdades existentes no acesso à justiça em matéria ambiental. Para tanto e mediante a realização do acampamento, foi feito contato com pessoas que residem em pequenos povoados e municípios rurais da província de Santa Fé e foi analisado o estado da problemática ambiental e os mecanismos existentes para peticionar em defesa dos direitos vulnerados. Na ocasião, foi abordada a experiência vivida na cidade de Santo Domingo, localizada no Departamento de Las Colonias. Relatam-se as tarefas realizadas, destacando-se os resultados obtidos nas pesquisas de opinião, entrevistas e atividades desenvolvidas durante o acampamento.

Palavras-chave: acesso à justiça; acampamentos; direito ambiental; riscos socioambientais; conflitos socioambientais.

Para citación de este artículo: Balaudo, C. G.; Franco, D. L.; Gazzo, M. V. y Rodríguez, A. (2021). Acampe por una justicia ecológica: una experiencia de extensión y acceso a la justicia en la localidad de Santo Domingo, provincia de Santa Fe. +E: Revista de Extensión Universitaria, 11(15), e0010. doi: 10.14409/extension.2021.15.Jul-Dic.e0010 


\section{Introducción}

El Proyecto de Extensión de Interés Social (PEIS) "Acampe por una justicia ecológica" se desarrolla desde finales del año 2019 en la Facultad de Ciencias Jurídicas y Sociales de la Universidad Nacional del Litoral (FCJS-UNL) y surgió a partir de la inquietud de integrantes del Proyecto de Investigación "Meulen: Renovación de aportes jurídicos sobre el problema ecológico" ${ }^{2}$ y de organizaciones no gubernamentales y actores sociales vinculados con el proyecto de investigación.

Meulen, que en lengua mapuche significa "remolino de viento", es un proyecto de investigación en ejecución en el Centro de Investigaciones de la FCJS-UNL cuyo objetivo consiste en realizar un aporte desde el derecho a la construcción y revisión de estrategias elaboradas en torno al problema ecológico con un enfoque sociojurídico que considere el contexto local y latinoamericano, así como su relevancia social y actualidad. A tales fines, el proyecto pretende realizar una revisión de los siguientes aspectos: (i) el estatuto jurídico reconocido a la naturaleza, la biodiversidad y los animales no humanos; (ii) el establecimiento de un diálogo entre la diversidad cultural y la diversidad biológica desde las diferentes cosmovisiones presentes en Latinoamérica; (iii) la articulación de las distintas disciplinas y saberes que se dedican a revisar el vínculo entre riesgos, ciencias y derecho; (iv) la urgente necesidad de construir un espacio solidario novedoso entre lo humano y no lo humano que posee su traducción en términos jurídicos.

El "problema ecológico" es entendido como un concepto que articula una multiplicidad de espacios y escalas que van desde lo global hasta lo local. En este último ámbito, divisamos una serie de dificultades que limitan y obstaculizan el acceso a la justicia a víctimas directas o indirectas del daño ambiental, en especial en localidades de menor densidad poblacional, donde sus propios habitantes recurren a la noción de "pueblo" como forma de identificación territorial.

Algunas de las problemáticas recurrentes que pudimos individualizar como resultado de la experiencia fueron: aplicación de herbicidas por medio de aspersiones o fumigaciones; contaminación agroindustrial de ríos, arroyos, bañados y otros ecosistemas; deforestación por medio de tala masiva e indiscriminada; degradación de la tierra; basurales a cielo abierto; daños contra la biodiversidad, entre otros temas con relevancia ambiental y sanitaria en la provincia de Santa Fe. A partir de esto, surgieron interrogantes concretos: ¿cuál es la posibilidad real de acceder a la justicia ante una fumigación ilegal? ¿De qué modo se puede solicitar el control sobre actividades contaminantes? ¿Cuáles son los principales problemas que aquejan a las personas que viven en esas localidades? ¿Hay diferencias con las grandes urbes cuando se trata de hacer un reclamo administrativo o judicial?

En la búsqueda de una doble transformación, la transformación social y la de la propia Universidad (Erreguerena, Nieto y Tommasino, 2020), configuramos un proyecto de extensión que articula un enfoque de derechos con la realidad social e institucional de distintos territorios de la provincia en materia de acceso a la justicia. El proyecto se pensó con el objetivo de contribuir a una organización interna y territorial de vecinos y vecinas, hasta el momento ausente o incipiente, a los fines de lograr mayor autonomía al momento de realizar

1) El Proyecto fue aprobado por Resolución $N^{\circ} 122$ del Consejo Superior de la UNL. 2) Dirigido por la Prof. María Valeria Berros. 
reclamos, talleres y capacitaciones, plantear acciones ante la justicia o bien idear normativas sobre los problemas ambientales que afectan la vida cotidiana de las personas.

El mismo contempla como innovación metodológica una construcción colectiva y participativa sobre los problemas socioambientales existentes en las distintas zonas geográficas. A través de los acampes, surgió la propuesta de articular el campo del derecho (en particular, el derecho ambiental, los derechos de la naturaleza y los derechos humanos) con otras áreas del conocimiento (medicina, ciencias agrarias, ambientales y de la conservación). Esto último fue posible gracias a la interdisciplinariedad del equipo extensionista y la participación de diversas instituciones y organizaciones, privilegiando los saberes y experiencias mediante un intercambio dialógico, participativo y horizontal.

El marco geográfico seleccionado para desarrollar las actividades de extensión universitaria comprende a las localidades de Humboldt y Santo Domingo, ubicadas en el departamento Las Colonias de la provincia de Santa Fe. La pandemia por COVID-19 impidió llevar a cabo el acampe en la primera de aquellas, es por eso que en este texto recuperamos la experiencia transitada en Santo Domingo en el mes de septiembre del año 2019.

Para ello, organizamos el artículo en dos apartados. El primero intenta dar cuenta de las herramientas jurídicas disponibles en materia de acceso a la justicia ambiental en nuestro país, mientras que el segundo efectúa un relato de las actividades realizadas en la localidad y brinda los resultados de las encuestas elaboradas para, finalmente, establecer una serie de conclusiones.

\section{Acceso a la justicia ecológica y territorios: ¿una agenda pendiente?}

La sociedad, de manera gradual, ha dejado de asumir determinados riesgos implícitos en actividades potencialmente nocivas para la salud y el ambiente y, frente a la proliferación de conflictos socioambientales, se organiza para dar respuestas que pueden ser identificadas como institucionales o administrativas, por un lado, y no institucionales o contenciosas, por otro (Christel, 2020). Esta segunda clasificación se torna relevante para el tema en desarrollo debido a que nos permite ver cómo la articulación entre la ciudadanía y las diferentes herramientas jurídicas que el derecho otorga para el acceso a la justicia constituye una vía central para alcanzar la tutela efectiva de derechos que se consideran vulnerados.

El acceso a la justicia ambiental se relaciona de modo directo con el reconocimiento de derechos ambientales. Desde la Conferencia de Estocolmo de 1972, la mayoría de los países del mundo ha reconocido el derecho a un ambiente sano y ha incorporado normas sustanciales y procedimentales ligadas a la justicia ambiental. A ello se suman iniciativas más recientes que, en las primeras décadas del siglo XXI, reconocieron a la naturaleza como sujeto de derechos (Berros, 2013). Esto abrió nuevos caminos para repensar el vínculo entre justicia y ambiente: mientras que la justicia ambiental se basa en el derecho humano a un ambiente sano, la reciente noción de justicia ecológica extiende el concepto de justicia a la naturaleza y sus componentes, incluyendo a otros seres vivos no humanos (Gudynas, 2010). También es relevante mencionar que existen tratados internacionales que regulan diferentes aspectos específicos de la cuestión ambiental. Sobre este último punto, recientemente entró en vigor el Acuerdo de Escazú, primer tratado vinculante para Latinoamérica y el Caribe que regula de manera expresa el acceso a la justicia en asuntos ambientales. 
En Argentina, la reforma constitucional de 1994 incorporó el derecho de todos los habitantes del país a un ambiente sano y equilibrado, constitucionalizó el daño ambiental y situó en cabeza de las autoridades el deber de proteger este derecho. A su vez, estableció la distribución de competencias entre la Nación y las provincias en materia ambiental: la Nación dicta leyes de presupuestos mínimos, los cuales no pueden ser desconocidos por las provincias. Los Estados provinciales pueden complementar lo establecido por las leyes de presupuestos mínimos, es decir, pueden avanzar hacia mayores niveles de protección. Además, las provincias conservan el dominio originario de los "recursos naturales" existentes en su territorio.

En el año 2002, el Congreso Nacional aprobó la Ley 25675, conocida como Ley General del Ambiente, que delinea la política ambiental en nuestro país. También es menester tener presente que en nuestro ordenamiento jurídico se pueden identificar otras leyes de presupuestos mínimos de protección ambiental sobre temáticas específicas, como lo son: Ley 25612 de la Gestión Integral de Residuos de Origen Industrial y de Actividades de Servicio; Ley 25670 de Gestión y Eliminación de PCBs; Ley 25688 de Gestión Ambiental de Aguas; Ley 25831 sobre el Régimen de Libre Acceso a la Información Pública Ambiental; Ley 25916 sobre Gestión de Residuos Domiciliarios; Ley 26331 de Protección de los Bosques Nativos; Ley 26562 de Control de Actividades de Quema; Ley 26639 de Preservación de Glaciares y del Ambiente Periglacial; Ley 26815 de Manejo del Fuego; Ley 27279 de Gestión de Envases Vacíos de Productos Fitosanitarios; Ley 27520 de Adaptación y Mitigación al Cambio Climático Global.

Por su parte, la provincia de Santa Fe incorporó instrumentos normativos como la Ley 11717 de Medio Ambiente y Desarrollo Sustentable, sancionada en el año 1999 y que entre sus objetivos presenta el de "asegurar el derecho irrenunciable de toda persona a gozar de un ambiente saludable, ecológicamente equilibrado y adecuado para el desarrollo de la vida y la dignidad del ser humano". Otras leyes refieren a actividades específicas, como la Ley provincial 11273 de Productos Fitosanitarios, que regula la "elaboración, formulación, transporte, almacenamiento, distribución, fraccionamiento, expendio, aplicación y destrucción de envases de productos fitosanitarios" (art. 2). En tanto, en materia de acceso a la justicia ambiental, Santa Fe cuenta con la Ley 10000 sobre Recurso Contencioso Administrativo Sumario para la tutela de intereses simples o difusos, entre los que se enumera la "conservación de la fauna y la flora" y la "protección del medio ambiente".

Cabe señalar, como mencionamos con antelación, que el 22 de abril de 2021 entró en vigor el Acuerdo Regional sobre el Acceso a la Información, la Participación Pública y el Acceso a la Justicia en Asuntos Ambientales en América Latina y el Caribe, celebrado en Escazú, República de Costa Rica y ratificado por Argentina con la Ley 27566. El mismo supone un hito en favor de la consolidación de las democracias ambientales, promueve la construcción de mayores niveles de confianza ante las decisiones adoptadas, estipula mayor prevención ante los conflictos socioambientales, y plantea una redefinición de las relaciones entre Estado, mercado y sociedad.

Este breve recorrido permite identificar la existencia de normas en diferentes escalas regulatorias que tienen la potencialidad de constituirse como herramientas jurídicas relevantes a la hora de garantizar un acceso efectivo a la justicia en materia ambiental. Este es un derecho humano y, si bien existen dificultades en su cumplimiento desde una perspectiva generalizada, en cuestiones ambientales los obstáculos aparentan ser mayores y se profun- 
dizan en las pequeñas localidades, principalmente porque las mismas enfrentan mayores obstáculos como consecuencia de la ubicación geográfica, actividades productivas y económicas que desarrollan y organización social e institucional.

\section{La experiencia en la localidad de Santo Domingo}

Como referimos al principio del presente artículo, el equipo extensionista identificó dos localidades en el departamento Las Colonias en las que existían problemáticas ambientales $y$, a la vez, en ellas se encontraba un conjunto de actores sociales e instituciones vinculado previamente con el Proyecto. De esta manera, se fue construyendo colectivamente la experiencia de los "acampes jurídicos"3 y se definió que Humboldt y Santo Domingo fueran los primeros lugares en los que se desarrollarían. Además de tratarse de localidades pequeñas, poseen algunos problemas en común; el más destacado de ellos se vincula con las fumigaciones que se realizan alrededor de sus ejidos urbanos desde hace años.

La primera localidad visitada fue Santo Domingo. Con anterioridad al acampe, se generó una instancia de planificación y organización en la que se diagramó el contenido de la encuesta que se haría a la población, se organizó el traslado y el hospedaje de las personas integrantes del equipo extensionista y se acordó una serie de actividades, entre las que estaban la realización de una radio abierta con niños, niñas y adolescentes, y la presentación de un protocolo de actuación ante casos de fumigaciones.

El equipo que llevó a cabo este primer acampe estuvo integrado por docentes, graduados y graduadas, así como por estudiantes de la FCJS-UNL. Participó también un grupo de voluntarios y voluntarias provenientes de otras unidades académicas, especialmente de la Facultad de Ingeniería Química (FIQ-UNL) y estudiantes de la carrera de Abogacía que realizaron parte de su Práctica Profesional Final en el marco del Proyecto de Extensión. Por último, también participaron instituciones y personas que residían en el momento en Santo Domingo ${ }^{4}$ y organizaciones de la sociedad civil con sede en la ciudad de Santa Fe. ${ }^{5}$

Es importante resaltar el rol que tuvieron las instituciones locales dado que el mismo fue primordial para generar un primer acercamiento con los vecinos y vecinas del lugar, destacándose el trabajo de sus representantes, reconocidos referentes de la localidad. Su aporte fue relevante para interiorizar a los vecinos acerca de las problemáticas ambientales que afectan a su pueblo así como para plantear en forma conjunta interrogantes y alternativas desde una mirada comprometida con la transformación de la realidad que habitan. Rafaghelli sostiene que: "Las relaciones intersubjetivas que se dan en la extensión universitaria, en tanto acción dialógica, posibilitan construir prácticas democráticas de comunicación y formación

3) Esta expresión se inspira en los campamentos sanitarios que se vienen desarrollando en la Facultad de Ciencias Médicas de la Universidad Nacional de Rosario bajo la coordinación de Damián Verzeñassi, responsable académico del ciclo de Práctica Final de Medicina. Puede consultarse un registro fotográfico de nueve años de campamentos sanitarios de autoría de Javier Albea (2020), editado por Damián Verzeñassi y publicado por el Instituto de Salud Socioambiental.

4) Sociedad Médica del Departamento Las Colonias (referente Dr. Arturo Serrano) y Asociación Civil IPSI N 4023 Los Colonizadores (referente: Vanina Cherri).

5) Las asociaciones civiles "Centro de Protección de la Naturaleza" y "Capibara. Naturaleza, Derecho y Sociedad" forman parte del Proyecto desde su formulación. 
entre los sujetos y las instituciones" (2013, p. 23). Esto permite comprender que el trabajo de extensión, en palabras de Celman, se presente como:

"Un proceso dialógico y bidireccional, que busca la colaboración entre actores universitarios y actores sociales, en un pie de igualdad, dialogando y combinando sus respectivos saberes al servicio de objetivos socialmente valiosos, dando prioridad a los problemas de los sectores más postergados". (2013, p. 10)

Esta heterogeneidad de miembros en el equipo posibilitó que el diseño de las actividades y la planificación se nutrieran de recorridos previos y de perspectivas disciplinares diversas. En un inicio, el instrumento principal de trabajo que se discutió en el seno del equipo fue la confección y diagramación de la encuesta que se aplicaría en la localidad. La misma se efectuó tras varias reuniones y contó con los aportes de expertos externos al grupo. Los temas centrales a abordar fueron los siguientes:

I. Estilo de vida y ambiente: esta primera parte tuvo como objetivo conocer la situación del ambiente en la localidad a través de la detección de diferentes problemáticas presentes en la vida cotidiana de sus habitantes.

II. Legislación: la segunda se enfocó en identificar el conocimiento de las vecinas y vecinos sobre los principales derechos de los que son titulares en materia ambiental, considerando especialmente el contenido constitucional desde 1994, entre otros.

III. Acceso a la justicia: la tercera parte pretendió detectar las instituciones y autoridades locales a las que acudirían quienes tomaran conocimiento o se vieran afectados por un conflicto ambiental.

IV. Información sociodemográfica: datos personales de cada encuestado, como su sexo, edad, profesión, situación laboral, formación académica y composición de su grupo familiar, excluyendo su nombre y apellido en razón del anonimato de la encuesta.

Una vez completados todos los preparativos, el 28 de septiembre de 2019 se llevó a cabo el primer acampe. Santo Domingo, que se encuentra a $80 \mathrm{~km}$ de la capital provincial, forma parte de la región santafesina que fue escenario de la radicación de las primeras colonias agrícolas a partir de la fundación de Esperanza ${ }^{6}$ en 1856, capital del departamento. Mantuvo el liderazgo provincial como productora cerealera por más de tres décadas y proveyó el núcleo básico alrededor del cual se organizó y se expandió el proceso de colonización agrícola (Gallo, 2007). Hülsberg, menciona que: "Su ubicación estratégica en una zona de pleno desarrollo, y sus tierras aptas para la agricultura, lograron una de las colonizaciones más exitosas de la zona" (2003, p. 965).

Actualmente, la región cuenta con un gran desarrollo agropecuario, potenciado en los últimos años por la explotación tambera y el sector industrial.

Según el último Censo nacional del año 2010, habitan en el pueblo 1742 personas. Teniendo presente estas características y la historia del lugar, emprendimos la realización de las encuestas, los integrantes del equipo se dividieron en parejas que fueron caminando por casi la totalidad de las manzanas que componen la localidad.

6) Ciudad del centro-este de la provincia de Santa Fe en la República Argentina. Se ubica a $38 \mathrm{~km}$ de la ciudad de Santa Fe por ruta y a $30 \mathrm{~km}$ en línea recta. 


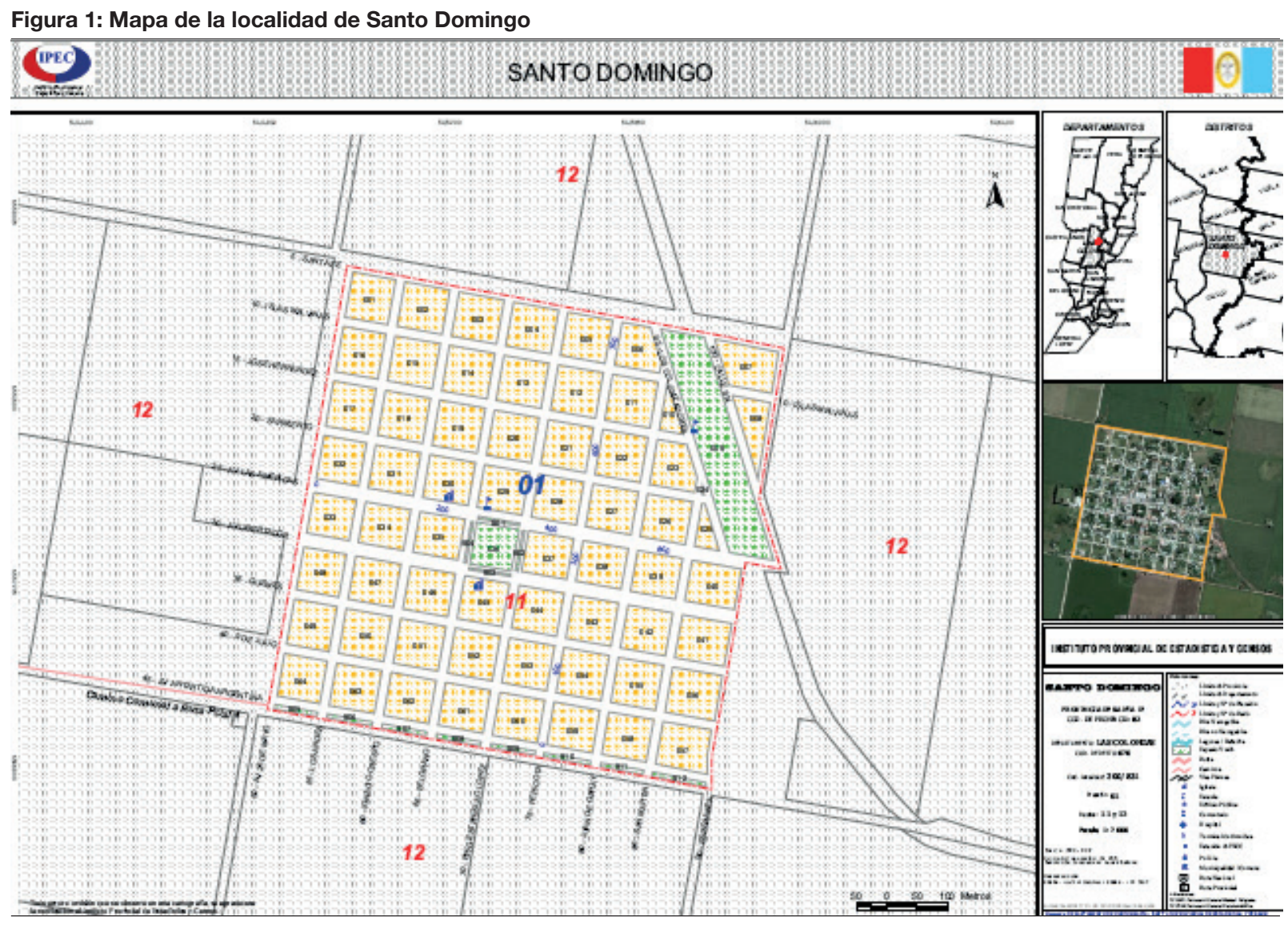

Fuente: Instituto Provincial de Estadística y Censos (IPEC).

Durante el recorrido y la realización de las encuestas, los vecinos y vecinas recibieron amablemente al equipo en sus hogares. Muchas de las personas estaban al tanto de la existencia del Proyecto y de las actividades que el mismo pretendía llevar a cabo en la localidad, esto fue así debido al trazado previo de una estrategia comunicacional: los referentes de las instituciones locales informaron por radio respecto de la visita del equipo, el contenido y los objetivos del Proyecto. También se entregaron comunicados a los alumnos de instituciones educativas del lugar para que las familias pudieran informarse sobre los puntos anteriormente mencionados.

La agenda del Proyecto involucró, además de las encuestas, distintas entrevistas a personas destacadas por su labor en la región con la finalidad de conocer su modo de ver la situación ambiental y el acceso a la justicia ante la presencia de conflictos vinculados a este tema en la localidad. Entre las personas entrevistadas, podemos mencionar a Patricia Arber (presidenta comunal), Vanina Cherri (docente), Arturo Serrano (médico), y Hugo Bidart (director de la Escuela Agrotécnica Los Colonizadores).

A partir de estas entrevistas, pudimos analizar el trabajo del Poder Ejecutivo local, observar cómo eran abordados los conflictos ambientales y la forma en que estos eran percibidos por distintos actores desde la óptica de sus profesiones. 
El día domingo 29 de septiembre, luego de la jornada de encuestas puerta a puerta, se efectuó una actividad colectiva y de libre acceso en la plaza central, la llamada "Merienda por el ambiente". Planteamos como parte del encuentro diferentes actividades en simultáneo, juegos y radio abierta para niños, niñas y adolescentes, y una charla con un grupo de vecinos y vecinas donde expresaron su visión acerca de la situación ambiental del pueblo. También participaron de esta instancia personas domiciliadas en localidades aledañas.

En esa ronda, los participantes se explayaron acerca de cómo se veían afectados por los problemas ambientales que se suscitaban en su entorno. El encuentro fue valorado por todos los presentes y significó un momento de reunión en donde compartieron información y opiniones sobre sus propias vivencias. A pesar de conocerse, nunca habían tenido una instancia de diálogo colectivo para expresarse y debatir ideas sobre problemáticas en común.

En esta última actividad, y a modo de cierre del campamento, el equipo extensionista presentó un Protocolo de Actuación ante casos de fumigaciones. Elaboramos el mismo con la finalidad de contribuir con una herramienta capaz de generar mejoras en materia de acceso a la justicia ambiental. En su texto, destacamos la importancia de registrar el momento en que se efectúa una fumigación, con la premisa de que toda la información que pueda recolectarse resulta fundamental como prueba del hecho para una posterior denuncia. Incluimos también descripciones de las distintas vías para realizar una denuncia o reclamo formal ante las autoridades municipales o provinciales competentes.

Como resultado final del primer acampe, se obtuvieron 158 encuestas que fueron sistematizas por estudiantes de la Práctica Profesional Final de la carrera de Abogacía de la UNL y se pudo elaborar un informe con obtuvieron datos relevantes sobre el acceso a la justicia en materia ambiental en la localidad de Santo Domingo.

En primer lugar, el 68,4 \% de las personas encuestadas consideró el estado del ambiente como bueno, sin embargo, al momento de identificar conflictos ambientales, la mayoría de las respuestas indicó que las fumigaciones y los productos químicos utilizados para realizar las mismas eran un problema frecuente en la localidad. La contaminación del agua también fue mencionada como un tema de importante.

En relación con las fumigaciones, a partir del relato de las personas se pudieron identificar casos concretos de vecinos y vecinas que padecían y/o habían padecido dificultades en la salud como consecuencia de estas prácticas agrícolas. El caso de un joven que trabajaba en la manipulación de agrotóxicos fue el que más resonó. Era conocido por todos los habitantes de la localidad, quienes habían notado el deterioro en su salud hasta su fallecimiento.

Otro dato a destacar fue que los vecinos y vecinas indicaron que era habitual la realización de fumigaciones con herbicidas sobre el césped para evitar su crecimiento en domicilios particulares. A causa de ello y preocupados por su salud, en muchas oportunidades optaban por recluirse en sus hogares para no sufrir afectaciones.

Estas problemáticas, según la opinión mayoritaria de los encuestados, deberían regularse y no prohibirse. En este sentido, manifestaron que una buena regulación permitiría su desarrollo y reduciría los efectos nocivos, pero no consideraron la prohibición ya que, por ejemplo, varios vecinos y vecinas trabajaban en la actividad agropecuaria, la cual es primordial para la economía del pueblo. 
Figura 2: Resultado de encuestas sobre el estado del ambiente en la comunidad ¿Cuál es el estado del ambiente para usted en la comunidad?

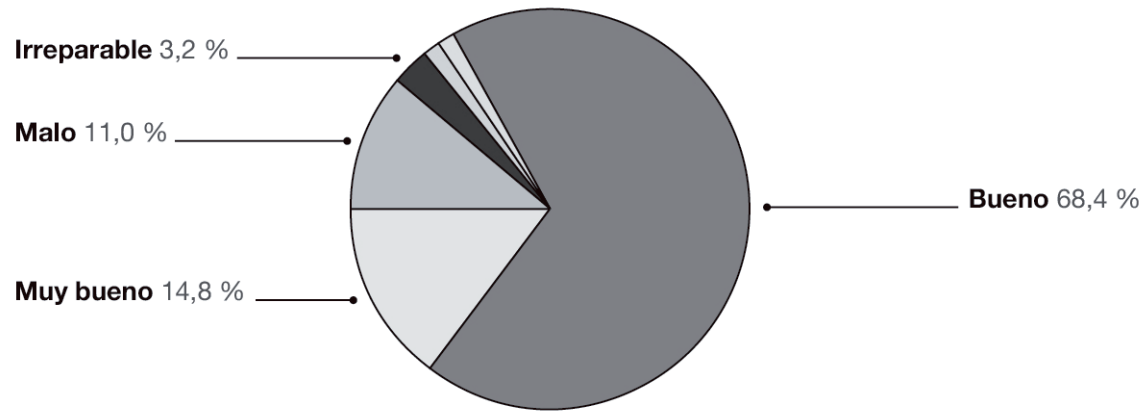

Fuente: PEIS "Acampe por una justicia ecológica".

Figura 3: Resultado de encuestas sobre responsabilidad por contaminación

Si una actividad pudiera generar contaminación:

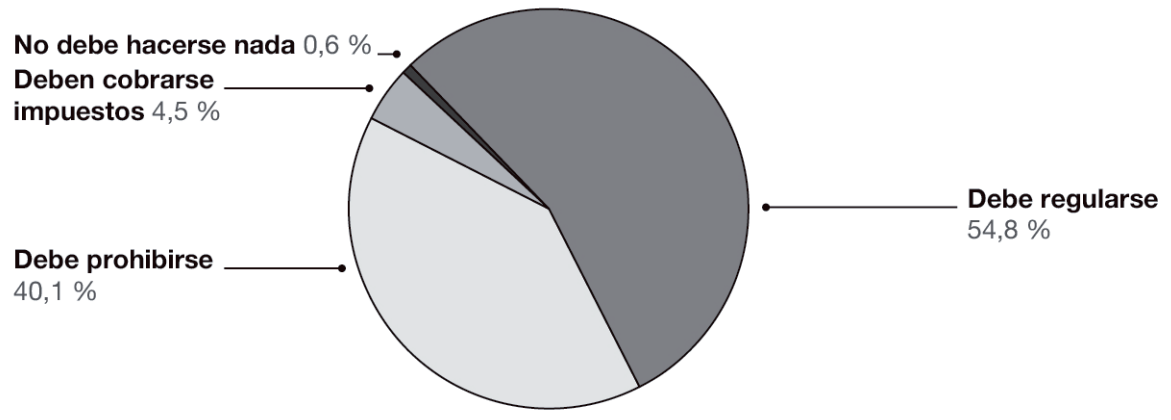

Fuente: PEIS "Acampe por una justicia ecológica".

Sobre la sección destinada a legislación, un 63,6 \% de la población desconocía que la Constitución Nacional contiene un artículo que reconoce el derecho de todos los habitantes a un ambiente sano. Un porcentaje similar al anterior indicó tener conocimiento sobre la existencia de otras normas protectorias, sin embargo, no pudieron identificar ningún ejemplo.

Resulta interesante mencionar que en el apartado de la encuesta dedicado a legislación protectoria del ambiente, quienes desconocían la existencia de normativa se sorprendían y consultaban de forma inmediata por su efectividad y aplicación en la práctica. 
Figura 4: Resultado de encuestas sobre derecho al ambiente sano en la Constitución Nacional

¿Sabe usted que la Constitución Nacional reconoce el derecho de todos los habitantes a un ambiente sano?

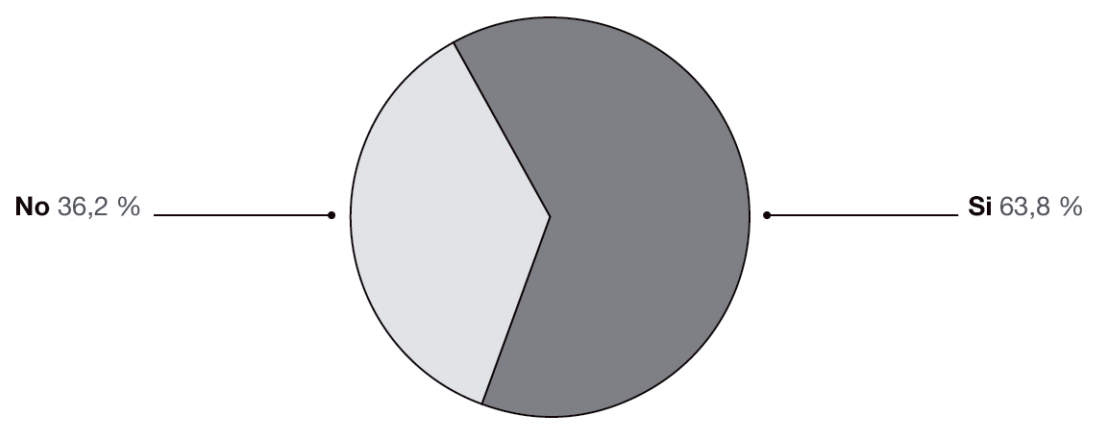

Fuente: PEIS "Acampe por una justicia ecológica”.

Figura 5: Resultado de encuestas sobre legislación protectoria del ambiente

¿Sabe que existe legislación protectoria del ambiente?

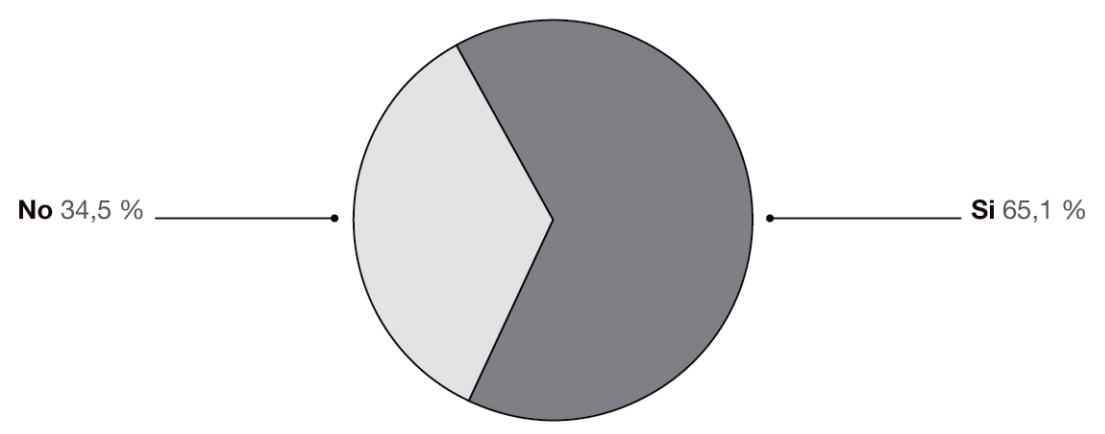

Fuente: PEIS "Acampe por una justicia ecológica”.

En caso de que ocurra un conflicto ambiental, las personas encuestadas establecieron que, si como consecuencia de la contaminación se produjeran afectaciones a la salud, alguien debería asumir la responsabilidad. En ese sentido, se identificó como responsables a quienes generan la contaminación. 
Figura 6: Resultado de encuestas con referencia a la responsabilidad por contaminación

¿Usted cree que si la contaminación genera problemas de salud debe haber responsables?

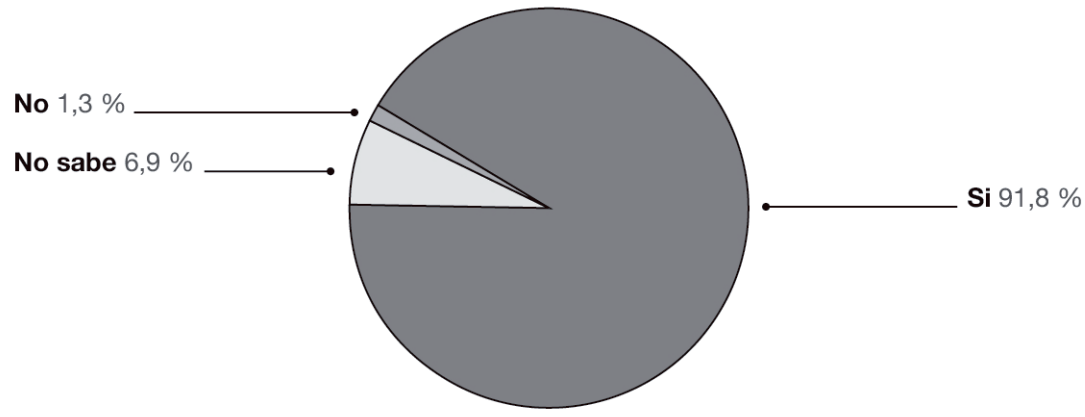

Fuente: PEIS "Acampe por una justicia ecológica".

Al momento de determinar instituciones y personas que pudieran brindar asistencia frente a estos conflictos se dieron diversas respuestas. Entre ellas, se identificó a la Comuna de Santo Domingo (66,1\%), hospital/médicos (8,3\%), policía (5\%), Greenpeace (3,3 \%) y hubo un amplio porcentaje de encuestados que desconocía quiénes podrían asistirlos (15,7 \%).

Figura 7: Resultado de encuestas sobre instituciones que pueden brindar asistencia frente a un conflicto ambiental

¿Qué institución cree que puede ayudarle? Indíquela

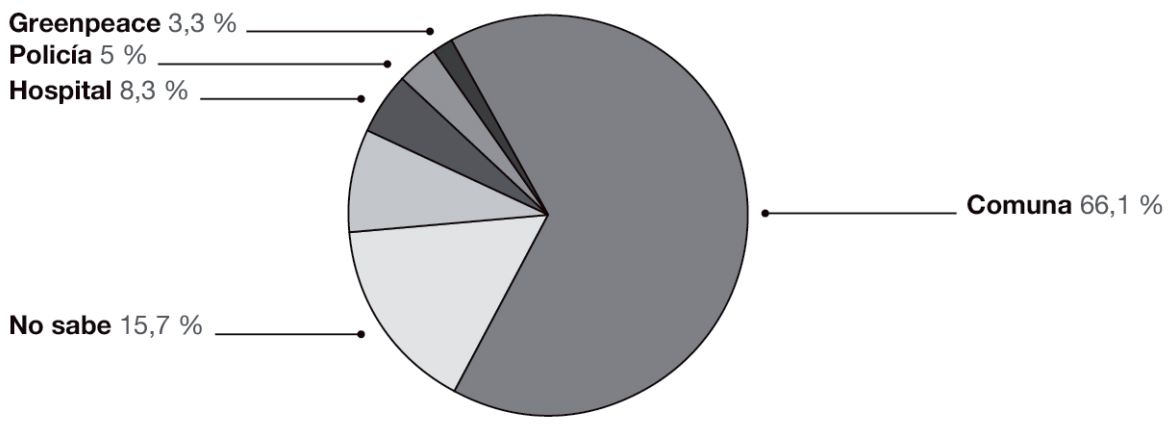

Fuente: PEIS "Acampe por una justicia ecológica".

A partir de esta pregunta con respuestas variadas se pudo relevar la ausencia de información específica sobre instituciones capaces de dar asistencia ante un conflicto socioambiental. Si bien la mayoría de las personas identificó a la Comuna como la institución a la cual acudirían en primer lugar, es importante tener en cuenta que hay un gran número que respondió no saber a dónde acudir. A modo anecdótico, un vecino, al responder esta pregunta, manifestó que "sus amigos, los muchachos del pueblo, podrían ayudarlo". 
Por otro lado, es relevante destacar que el 83,3 \% respondió que nunca había realizado una denuncia sobre las problemáticas ambientales existentes. En tanto, quienes habían efectuado denuncias, comentaron que las respuestas recibidas por las personas e instituciones a las que habían acudido no fueron satisfactorias.

\section{Figura 8: Resultado de encuestas sobre denuncias por problemáticas ambientales}

\section{¿Alguna vez denunció este tipo de problemáticas?}

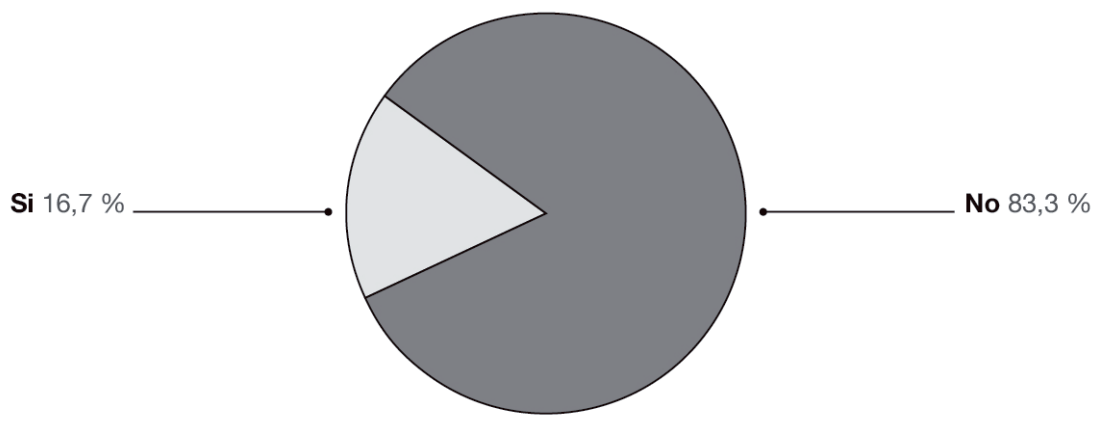

Fuente: PEIS "Acampe por una justicia ecológica”.

Figura 9: Resultado de encuestas sobre respuestas institucionales recibidas frente a la realización de denuncias por problemáticas ambientales

¿Fue satisfactoria la respuesta?

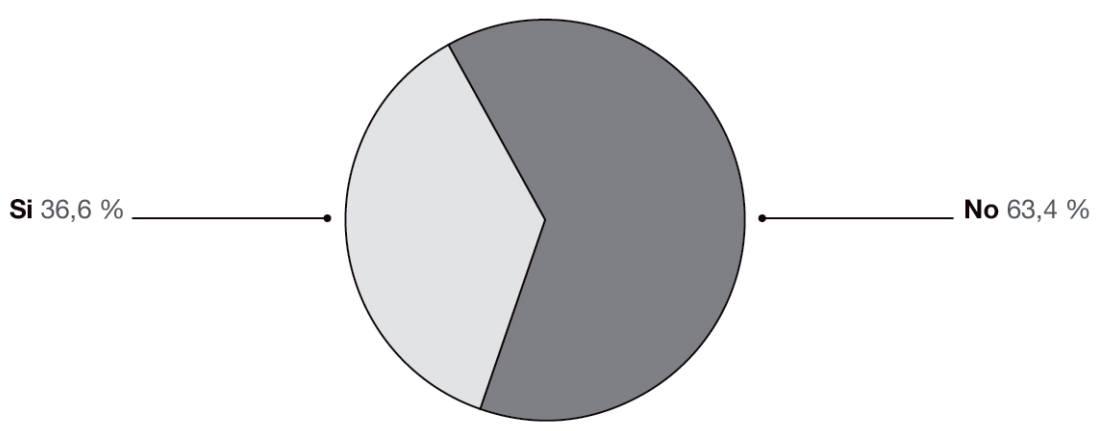

Fuente: PEIS “Acampe por una justicia ecológica”.

Por último, respecto de la pregunta sobre qué sería necesario para proteger el ambiente, el $30 \%$ de las personas encuestadas consideró que lo era generar conciencia y educación entre la población. Al mismo tiempo, fueron seleccionadas en menor porcentaje otras opciones tales como: la decisión política, la información y el dinero. 
Figura 10: Resultado de encuestas sobre protección del ambiente en la localidad ¿Qué hace falta para proteger el ambiente en su localidad?

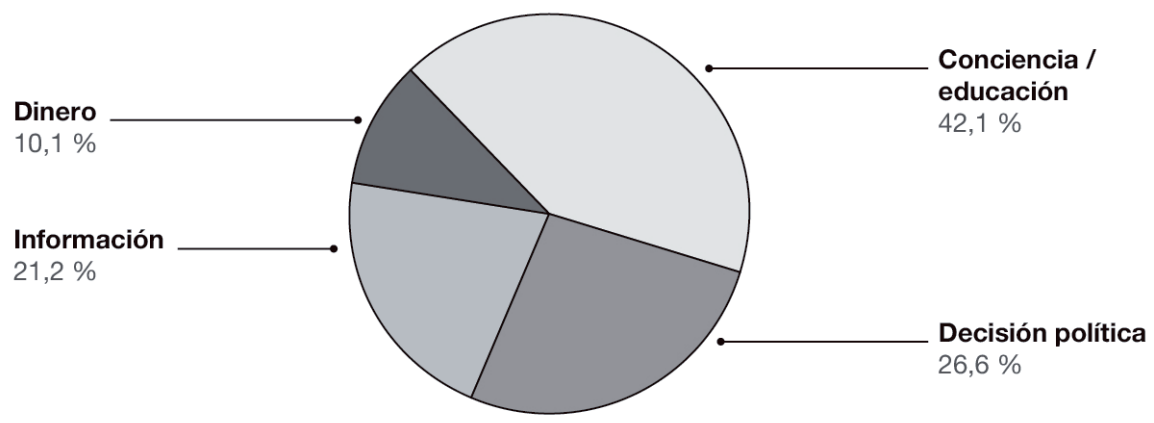

Fuente: PEIS "Acampe por una justicia ecológica".

\section{Conclusiones}

Los modos de interacción que se producen en el contexto social son fundamentales para comprender el carácter comunitario del conocimiento y la importancia que en todo proceso de formación tienen el reconocimiento y el respeto hacia los otros. Entendemos la extensión universitaria como espacio de prácticas sociales compartidas y colaborativas para la construcción de propuestas alternativas que permitan intervenir de manera creativa e innovadora en las situaciones identificadas como problemas sociales.

La primera experiencia de acampe jurídico en Santo Domingo posibilitó individualizar aquellas situaciones problemáticas que se presentan en el pueblo a través del relato de las personas que participaron de las diferentes actividades desarrolladas por el equipo extensionista y de los datos relevados en las encuestas y entrevistas. De los aportes suministrados, pudimos observar que existe aceptación en cuanto a concebir al ambiente como algo fundamental y llevar adelante prácticas para su cuidado. Sin embargo, la mayoría de los lugareños desconocía la legislación protectoria.

Advertimos también la escasa iniciativa de la comunidad para efectuar una denuncia ante un problema ambiental sustentada en la idiosincrasia del lugar — propia de una localidad pequeña, donde quienes la habitan se conocen entre sí y resulta muy incómodo acusarse entre allegados- y por la naturaleza agraria de su actividad económica principal, fuente de sustento de las familias de la región. Pero, también, por no saber de qué manera proceder, a dónde acudir y ante quién peticionar frente a un conflicto de esta naturaleza.

Lo mencionado representa un gran obstáculo para poder acceder al derecho a un ambiente sano. Sin embargo, entendemos que eso puede ser subsanado si hay una incorporación y puesta en práctica de la información brindada, dado que no existe una naturalización de las conductas que perjudican el ambiente sino, por el contrario, están identificadas y son una gran preocupación para los miembros de la comunidad.

De la reunión en la plaza surgieron interrogantes y necesidades, pudimos brindar herramientas y generar acceso a la información sobre los derechos existentes y su forma de ejercerlos sin perder de vista las miradas de los pobladores. El entusiasmo percibido tras 
las jornadas de trabajo hizo posible que continuáramos en contacto con los referentes del lugar, comprometiéndonos a volver una vez que las condiciones sanitarias lo permitieran, para reflejar en un encuentro los resultados del acampe y juntos seguir repensando, problematizando, concientizando y reflexionando sobre la realidad ambiental de Santo Domingo.

\section{Referencias bibliográficas}

Celman, S. (2013). La extensión como espacio político y pedagógico. Prólogo. En Menéndez, G.; Camilloni, A. de; Rafaghelli, M.; Kessler, M. E.; Boffelli, M.; Sordo, S.; Pellegrino, E.; Malano, D. Integración, docencia y extensión. Otra forma de aprender y de enseñar. 1ra. ed. Ediciones UNL.

Christel, L. (2020). Protesta y estrategias legales ¿antagónicas o complementarias? El caso de la Asamblea El Algarrobo y su lucha contra la minería a cielo abierto. Revista Electrónica. Instituto de Investigaciones Ambrosio L. Gioja, (24).

Erreguerena, F.; Nieto, G. y Tommasino, H. (2020). Tradiciones y matrices, pasadas y presentes, que confluyen en la Extensión Crítica Latinoamericana y Caribeña. Cuadernos de Extensión Universitaria de la UNLPam, 4(4), 177-204.

Gallo, E. (2007). Colonos en armas: las revoluciones radicales en la provincia de Santa Fe (1893). 1ra. ed. Siglo XXI Editores.

Gudynas, E. (2010). La senda biocéntrica: valores intrínsecos, derechos de la naturaleza y justicia ecológica. Tabula Rasa, (13), 45-71.

Hülsberg, H. A. (2003). Historia de Progreso. Nuestro Pueblo Santafesino. Cogtal Talleres Gráficos.

Rafaghelli, M. (2013). La dimensión pedagógica de la extensión (pp. 22-38). En Menéndez, G.; Camilloni, A. de; Rafaghelli, M.; Kessler, M. E.; Boffelli, M.; Sordo, S.; Pellegrino, E.; Malano, D. Integración, docencia y extensión. Otra forma de aprender y de enseñar. 1ra. ed. Ediciones UNL. 